\title{
EXPRESSION PATTERN OF ODONTOGENIC AMELOBLAST-ASSOCIATED AND AMELOTIN DURING FORMATION AND REGENERATION OF THE JUNCTIONAL EPITHELIUM
}

\author{
Clarice Nishio ${ }^{1}$, Rima Wazen ${ }^{1}$, Shingo Kuroda ${ }^{1}$, Pierre Moffatt ${ }^{2}$, and Antonio Nanci ${ }^{*}$ \\ ${ }^{1}$ Laboratory for the Study of Calcified Tissues and Biomaterials, Department of Stomatology, Faculty of Dentistry, \\ Université de Montréal, Montréal, Québec, Canada \\ ${ }^{2}$ Shriners Hospital for Children, Montréal, Québec, Canada
}

\begin{abstract}
The junctional epithelium (JE) adheres to the tooth surface, and seals off periodontal tissues from the oral environment. This incompletely differentiated epithelium is formed initially by the fusion of the reduced enamel organ with the oral epithelium (OE). Two proteins, odontogenic ameloblast-associated (ODAM) and amelotin (AMTN), have been identified in the JE. The objective of this study was to evaluate their expression pattern during formation and regeneration of the JE. Cytokeratin 14 was used as a differentiation marker for oral epithelial cells, and Ki67 for cell proliferation. Immunohistochemistry was carried out on erupting rat molars, and in regenerating JE following gingivectomy. In the reducing enamel organ and in established JE, ODAM and AMTN were present at the celltooth interface while only ODAM and CK14 were found throughout the JE. Both were also conspicuously present in cell clusters situated between the erupting tooth and OE. During JE regeneration, ODAM was detected first at the leading wound edge and then in the regenerating JE. Some cell clusters in the subjacent connective tissue were also positive for ODAM. AMTN appeared later and both AMTN and ODAM accumulated at the interface with the tooth. Cytokeratin 14 gradually appeared in the regenerating JE but the cell clusters showed variable labeling. Cells associated with JE formation and regeneration exhibited higher division activity than adjacent epithelial cells. These findings suggest that ODAM and AMTN have a role at the cell-tooth interface, and that ODAM is likely also implicated in cellular events during formation and regeneration of the JE.
\end{abstract}

Keywords: Apin, amelotin, cytokeratin 14, development, gingival, junctional epithelium, ODAM, regeneration.

\footnotetext{
* Address for correspondence:

Antonio Nanci

Laboratory for the Study of Calcified Tissues and Biomaterials

Department of Stomatology, Faculty of Dentistry, Université de Montréal

2900 Edouard-Montpetit, Pavillon Roger-Gaudry, Room A-212, H3T1J4

Montréal, Québec, Canada

Telephone Number: +1514.3435846

FAX Number: +1514.3432233

E-mail: antonio.nanci@umontreal.ca
}

The junctional epithelium (JE) is a specialized epithelial structure that seals off the supporting tissues of the tooth from the aggressive oral environment, and represents the first line of defense against periodontal diseases (Takata et al., 1986; Schroeder and Listgarten, 1997; Schroeder and Listgarten, 2003). This stratified squamous, nonkeratinizing epithelium is considered to be incompletely differentiated, producing components for tooth attachment instead of progressing along a keratinization pathway (Schroeder and Listgarten, 2003; Shimono et al., 2003). The attachment of the gingiva to the enamel surface is provided by a structural complex called the epithelial attachment consisting of an inner basal lamina (BL) and hemidesmosomes. The BL is formed and maintained by the superficial JE cells to which they are attached by hemidesmosomes (Hormia et al., 1992; Hormia et al., 2001; Masaoka et al., 2009; Tsuchiya et al., 2009). The $\mathrm{BL}$ of the JE is atypical because it contains laminin-5 but not other typical components, such as $\gamma 1$ chain-containing laminins, and type IV and VII collagens (Hormia et al., 2001). However, the exact composition and attachment mechanism of the BL to the tooth surface are still not completely understood.

It is believed that during tooth development, the primary JE forms by the fusion of the reduced enamel organ with the oral epithelium (OE), and will be replaced in time by a secondary JE derived solely from cells of the OE (Sabag et al., 1984; Abe et al., 1995; Schroeder, 1996; Shimono et al., 2003; Bosshardt and Lang, 2005). Although the structure and the function of the JE are influenced by the underlying connective tissue (Mackenzie, 1987) and by contact with a solid substratum, such as enamel, dentin or cementum (Abe et al., 1995; Schroeder, 1996), the exact mechanisms that lead to the formation and regeneration of the JE remain unclear.

Efforts to identify the secretome of the epithelial cells responsible for creating tooth enamel (Moffatt et al., 2006a) have led to the identification of two genes encoding for secreted proteins called amelotin (AMTN) (Moffatt et al., 2006b) and APIN (Moffatt et al., 2008), APIN now being called odontogenic ameloblast-associated (ODAM). ODAM (Moffatt et al., 2006a; Moffatt et al., 2008) and AMTN (Iwasaki et al., 2005; Moffatt et al., 2006a; Moffatt et al., 2006b) are encoded by two different genes classified on the basis of their genomic location and architecture as part of the secretory calcium-binding phosphoprotein (SCPP) gene cluster (Kawasaki and Weiss, 2003; Huq et al., 2005; Kawasaki and Weiss, 2006). This cluster contains a number of genes coding for proteins that 
stabilize $\mathrm{Ca}$ and $\mathrm{PO}_{4}$ ions in body fluids and/or guide $\mathrm{CaPO}_{4}$ deposition into receptive extracellular matrices (Huq et al., 2005; Kawasaki, 2009). Interestingly, ODAM expression is highly upregulated in a number of epithelial cancers whose tissues of origin do not produce the protein (Solomon et al., 2003; Aung et al., 2006; Kestler et al., 2008), and has been suggested as a biomarker for human breast cancer (Siddiqui et al., 2009).

Broad expression profiling revealed that both ODAM (Park et al., 2007; Moffatt et al., 2008) and AMTN (Iwasaki et al., 2005; Moffatt et al., 2006b) are produced by ameloblasts during the maturation stage of amelogenesis. Unexpectedly, they are also strongly expressed in the JE (Moffatt et al., 2006b; Moffatt et al., 2008). However, these two proteins show different distribution patterns in the established JE. Both AMTN and ODAM are detected at the cell-tooth interface where the BL is found, but only ODAM is immunodetected throughout the cell layers of the JE. However, their pattern of expression during JE development and regeneration has so far not been established in details. In particular, the implication of ODAM and AMTN in the regenerating secondary JE is not known.

The objective of this study was therefore to investigate the presence and distribution of ODAM and AMTN at various stages of tooth eruption and in regenerating JE following gingivectomy. The labeling pattern was compared to that of cytokeratin 14 (CK14), a marker for JE and basal cells of OE (Hormia et al., 2001; Tsuchiya et al., 2009). The proliferation activity of cells implicated in JE development and regeneration was also examined.

\section{Materials and Methods}

\section{Development of the primary junctional epithelium}

Formation of the primary JE was followed in erupting maxillary molars of male Wistar rats (Charles River Canada, St Constant, QC, Canada) of 2 weeks (body weight $25 \pm 0.5 \mathrm{~g}), 3$ weeks $(50 \pm 0.5 \mathrm{~g})$ and 8 weeks of age (250 $\pm 0.5 \mathrm{~g})$. Each age group comprised 3 animals.

\section{Regeneration of the junctional epithelium}

Thirty adult male Wistar rats weighing $250 \pm 50 \mathrm{~g}$ (Charles River Canada) were used for this study. Animals were anesthetized with an intraperitoneal injection of a mixture of $50 \mathrm{mg} / \mathrm{kg}$ of ketamine hydrochloride $\left(\right.$ Ketaset $^{\circledR}$, Wyeth Canada, St-Laurent, QC, Canada), $5 \mathrm{mg} / \mathrm{kg}$ of xylazine (Rompun $^{\circledR}$, Bayer Inc., Toronto, ON, Canada), $1 \mathrm{mg} / \mathrm{kg}$ of acepromazine maleate (Acevet 10, Vétoquinol, Lavaltrie, QC, Canada) in distilled water. Removal of the gingiva and the JE along the maxillary molars (gingivectomy) was accomplished by scraping the tooth surface and extending $2 \mathrm{~mm}$ along the palate with periodontal curettes (SU 15/ 33, SL 5/6 and KS 1/2; Hi-Friedy, Chicago, IL, USA) (Nishio et al., 2010). Gingivectomies were carried out on the left side of maxilla and the contralateral side was left intact as control. Following surgery animals received a single injection of buprenorphine hydrochloride (Temgesic ${ }^{\circledR}$, Reckitt and Colman, Hull, UK). Six rats were sacrificed immediately after gingivectomy (day 0 ) and at $3,5,7$ and 14 days post-surgery. The animal procedures were in accordance with the guidelines of the Comité de déontologie de l'expérimentation sur les animaux of Université de Montréal.

\section{Tissue processing}

Animals were anesthetized with the above ketamine hydrochloride, xylazine and acepromazine maleate mixture, and sacrificed by intracardiac perfusion with a fixative solution consisting of 4\% paraformaldehyde and $0.1 \%$ glutaraldehyde in $0.08 \mathrm{M}$ sodium cacodylate buffer containing $0.05 \%$ calcium chloride, pH 7.2 (Nanci et al., 2004). Maxillae were dissected and immersed in the same fixative solution overnight at $4^{\circ} \mathrm{C}$. Maxillae from adult rats $(250 \mathrm{~g})$ were then decalcified for 30 days at $4^{\circ} \mathrm{C}$ in $4.13 \%$ disodium ethylenediamine tetraacetic acid (Warshawsky and Moore, 1967), and those from younger animals for 5 days, at $4^{\circ} \mathrm{C}$, in Planck-Rychlo solution consisting of $0.13 \mathrm{M}$ aluminium chloride hexahydrate (Sigma-Aldrich Canada Ltd, Oakville, Ont.), 0.2N hydrochloric acid (Fisher Scientific, Whitby, ON, Canada), $1.35 \%$ formic acid (Fisher Scientific) (Schroeder, 1991). Decalcified samples were washed for $24 \mathrm{~h}$ in $0.1 \mathrm{M}$ cacodylate buffer ( $\mathrm{pH} 7.2$ ), dehydrated through graded ethanols, cleared with xylene, embedded in paraffin and sectioned at $5 \mu \mathrm{m}$ thickness. Some sections were stained with hematoxylin and eosin for observation by light microscopy and others were processed for immunohistochemistry.

\section{Immunohistochemistry}

Sections were deparaffinized with Citrisolv (Fisher Scientific), rehydrated through a descending ethanol series and washed in distilled water. In order to avoid non-specific sticking, sections were blocked with 0.01 M PBS (pH 7.2) containing $5 \%$ skim milk for $20 \mathrm{~min}$ at room temperature. Incubations were carried out at room temperature with rabbit primary antibodies raised against rat ODAM $(1: 2,000,3 \mathrm{~h})$ (Moffatt et al., 2008) and AMTN (1:500, 3h) (Moffatt et al., 2006b) followed by a secondary goatanti-rabbit $(1: 500,1 \mathrm{~h})$ AlexaFluor 488 (for ODAM) or AlexaFluor 594 (for AMTN)-conjugated antibody (Invitrogen, Carlsbad, CA, USA). Following each incubation, slides were washed with $0.01 \mathrm{M}$ PBS containing $0.05 \%$ (v/v) Tween 20 (pH 7.4). Sections were mounted in ProLong Gold antifade reagent with 4',6Diamidine-2'-phenylindole dihydrochloride (DAPI) (Invitrogen) to counterstain nuclei in blue. Fluorescence was examined under a Carl Zeiss (Oberkochen, Germany) Axiophot microscope equipped with a DP70 Olympus (Tokyo, Japan) digital camera.

Immunoperoxidase labeling was used to detect CK14 and Ki67, a nuclear protein expressed during the active phases of the cell cycle (Endl and Gerdes, 2000). The sections were deparafnized, blocked and washed as described above, and then incubated with rabbit monoclonal primary antibody raised against human Ki67 (Clone SP6, 1:200, Thermo Fisher Scientific) and mouse monoclonal primary antibody raised against human CK14 

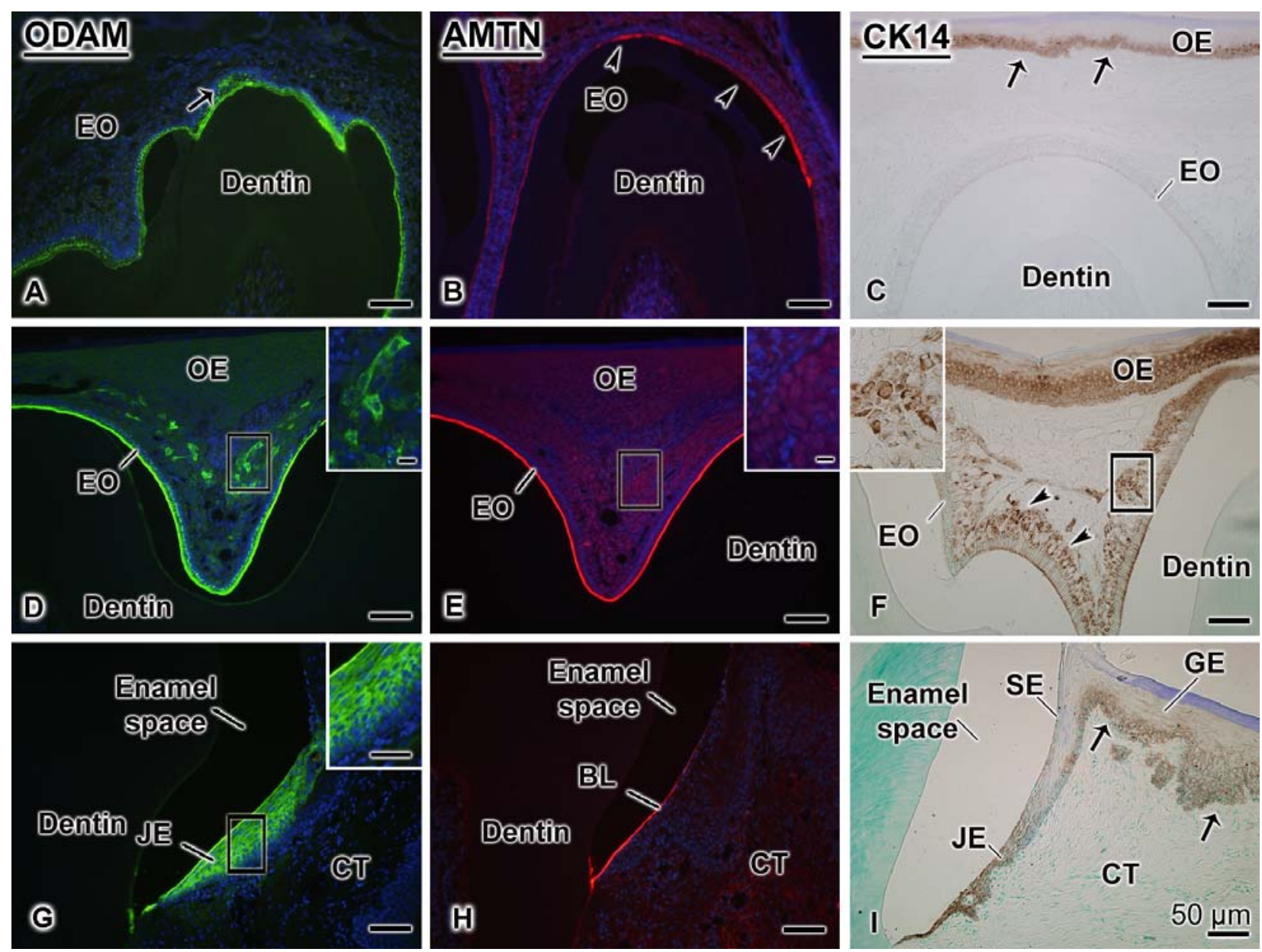

Fig. 1: Immunolabeling for ODAM (A,D,G), AMTN (B,E,H) and CK14 (C,F,I) in rats of 2 weeks (A-C), 3 weeks (D-F) and 8 weeks of age (G-I). At early stages of dental development, ODAM (arrow, A) was detected in the supranuclear region of reducing enamel organ (EO) cells. ODAM (A) was additionally immunodetected at the interface with the tooth. On the other hand, labeling for AMTN was restricted to the interface (arrowheads, B). CK14 (arrows, C) was detected in the basal cells of the oral epithelium (OE). Starting at 3 weeks, clusters of cells expressing ODAM (inset, D) and CK14 (inset, F) were observed between the reducing EO and OE. CK14 was also detected in cells of the papillary layer (arrowheads, F). (inset, G) In normal adult rats, labeling for ODAM was seen throughout the junctional epithelium (JE) as a pericellular labeling. Both ODAM (G) and AMTN (H) were detected at the JE-tooth interface where the inner basal lamina (BL) is found. (I) Immunolabeling for CK14 was observed in the gingival epithelium (GE) (arrows) and throughout the JE. SE, Sulcular epithelium; CT, connective tissue.

(1:500, R \& D Systems, Minneapolis, MN, USA) for $3 \mathrm{~h}$ at room temperature. The sections were treated with the DakoEnvision $^{\mathrm{TM}}+$ System, HRP labeled polymer antimouse (for CK14) and anti-rabbit (for Ki67) kits (Dako Corporation, Glostrup, Denmark) as recommended by the manufacturer. Visualization of the staining was performed with 3,3'-diaminobenzidine, and sections were then counterstained with $0.5 \%$ methyl green.

Immunolabeling of sections with pre-immune (Moffatt et al., 2006b; Moffatt et al., 2008), and omission of primary antibody were used as negative controls.

\section{Results}

Expression of ODAM, AMTN and CK14 during the development of the primary junctional epithelium In erupting first molars, at 2 weeks of age, the reducing enamel organ showed immunoreactivity for ODAM and AMTN but not CK14, which was restricted to the basal cells of the OE (Fig. 1A-C). Labeling for AMTN (Fig. 1B) was detected as a discrete line at the interface with the enamel surface. ODAM (Fig. 1A) was revealed in the supranuclear compartment of reducing ameloblasts and showed a more diffuse labeling at the interface. Starting with $1^{\text {st }}$ molar in 3 week postnatal rats and at later intervals for the other molars, there was strong immunoreactivity for ODAM (Fig. 1D) and CK14 (Fig. 1F), but not for AMTN (Fig. 1E), in cell clusters situated between the reducing enamel organ and the OE. CK14 was also distinctively present in the papillary layer of the reducing enamel organ (Fig. 1F) and widespread in the OE.

In 8-week-old rats in which all three molars were fullyerupted and the JE was well-established, both ODAM and AMTN were present at the interface with the tooth (Fig. $1 \mathrm{G}$ and $\mathrm{H})$. ODAM was additionally found among cells of the JE as a pericellular labeling (Fig. 1G). Immunolabeling for $\mathrm{CK} 14$ was intense in the gingival epithelium and throughout the JE (Fig. 1I). 

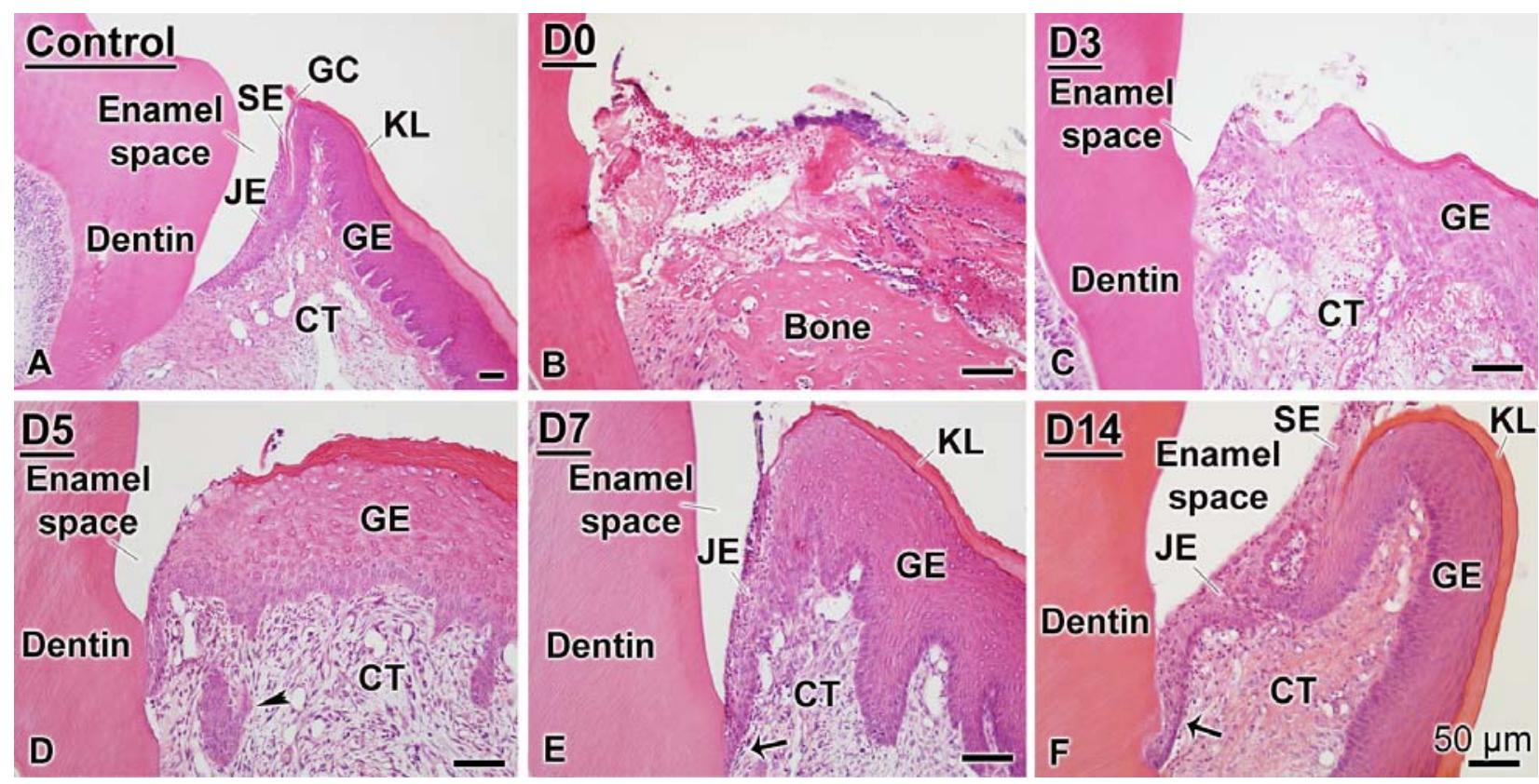

Fig. 2: Light microscopy of gingival wound healing following gingivectomy. (A) Micrograph of a control sample illustrating the normal organization of the dentogingival junction. The junctional epithelium (JE) attaches to the enamel surface. (B) Both the gingiva and the JE were removed by gingivectomy (D0). (C) At 3 days post-surgery (D3), the oral epithelium (OE) started to migrate towards the tooth. (D) At 5 days (D5), the reforming gingival epithelium (GE) was keratinized, and clusters of epithelial cells were observed at the cervical-most portion of the root (arrowhead). (E) By 7 days (D7), a regenerating long JE (arrow) was seen but the various portions of the dentogingival junction were not readily apparent. (F) At 14 days (D14), the long JE (arrow) extends down the root surface. CT, connective tissue; GC, gingival crest; SE, sulcular epithelium; KL, keratinized layer.

\begin{abstract}
Histology of regenerating junctional epithelium
In control samples, the normal dentogingival junction consisted of a sulcular epithelium and JE (Fig. 2A). Immediately after the gingivectomy (D0), there was blood exudation and formation of a fibrin clot at the gingival wound area (Fig. 2B). At day 3 post-surgery (D3), the OE had migrated towards the tooth (Fig. 2C). By day 5 (D5), a reforming gingival epithelium was apparent. It was keratinized, and clusters of epithelial cells were present in the underlying connective tissue (Fig. 2D). At day 7 after gingivectomy (D7), a JE was regenerating but there was no demarcation with the forming sulcular epithelium (Fig. 2E). At day 14 (D14), although the morphology of the newly formed gingival tissues was similar to control samples, the JE was long and extended down the tooth root (Fig. 2F).
\end{abstract}

\section{Expression of ODAM, AMTN and CK14 during the regeneration of the junctional epithelium}

After gingivectomy (D0), no labeling for ODAM (Fig. 3A), AMTN (Fig. 3B) and CK14 (Fig. 3C) was observed in the residual tissues. At D3, cells at the leading wound edge of the OE were labeled for ODAM (Fig. 3D). There was also staining at the interface with the tooth surface, and immunoreactive cell clusters were found in the subjacent connective tissue at the cervical-most portion of the root (Fig. 3D). No immunolabeling for AMTN (Fig. 3E) and CK14 (Fig. 3F) was noted on D3 at the wound site. At D5, in general, there was immunolabeling for ODAM at the tooth interface, among cells of the regenerating JE, and in cell clusters (Fig. 3G). These clusters showed no reactivity for AMTN and weak to intense labeling for CK14 (Figs. $3 \mathrm{H}$ and I). At this stage of wound healing, AMTN started to be immunodetected but only as an interfacial labeling between the cervical region of the tooth and regenerating JE (Fig. 3H). Cells of the reforming gingival epithelium showed intense CK14 labeling but those of the JE were only weakly reactive (Fig. 3I). By D7, expression of ODAM (Fig. 3J) was found throughout the cells of the long JE. Immunolabeling for CK14 resulted in an intense staining the gingival epithelium and a comparatively weak one in the JE (Fig. 3L). At D14, albeit for the presence of a long JE, the expression of ODAM (Fig. 3M) and AMTN (Fig. $3 \mathrm{~N}$ ) resembled that observed in the control 8-week old mouse samples (Fig. $1 \mathrm{G}$ and H). Distinctively at D14, immunolabeling for CK14 was now detected in the regenerated gingival epithelium, long JE and sulcular epithelium (Fig. 3O). Consistently, ODAM and AMTN were never detected in the gingival epithelium at all time points tested.

\section{Detection of cell proliferation activity during the formation and regeneration of the junctional epithelium}

As the reducing enamel organ fused with the OE to form the JE, abundant Ki67-labeled cells were observed on the outer layer of the enamel organ and in the basal layer of the OE (Fig. 4A and B). Once the tooth erupted and the JE was fully-formed, Ki67-immunolabeled cells in the gingival epithelium were not frequent (Fig. 4C) and the 


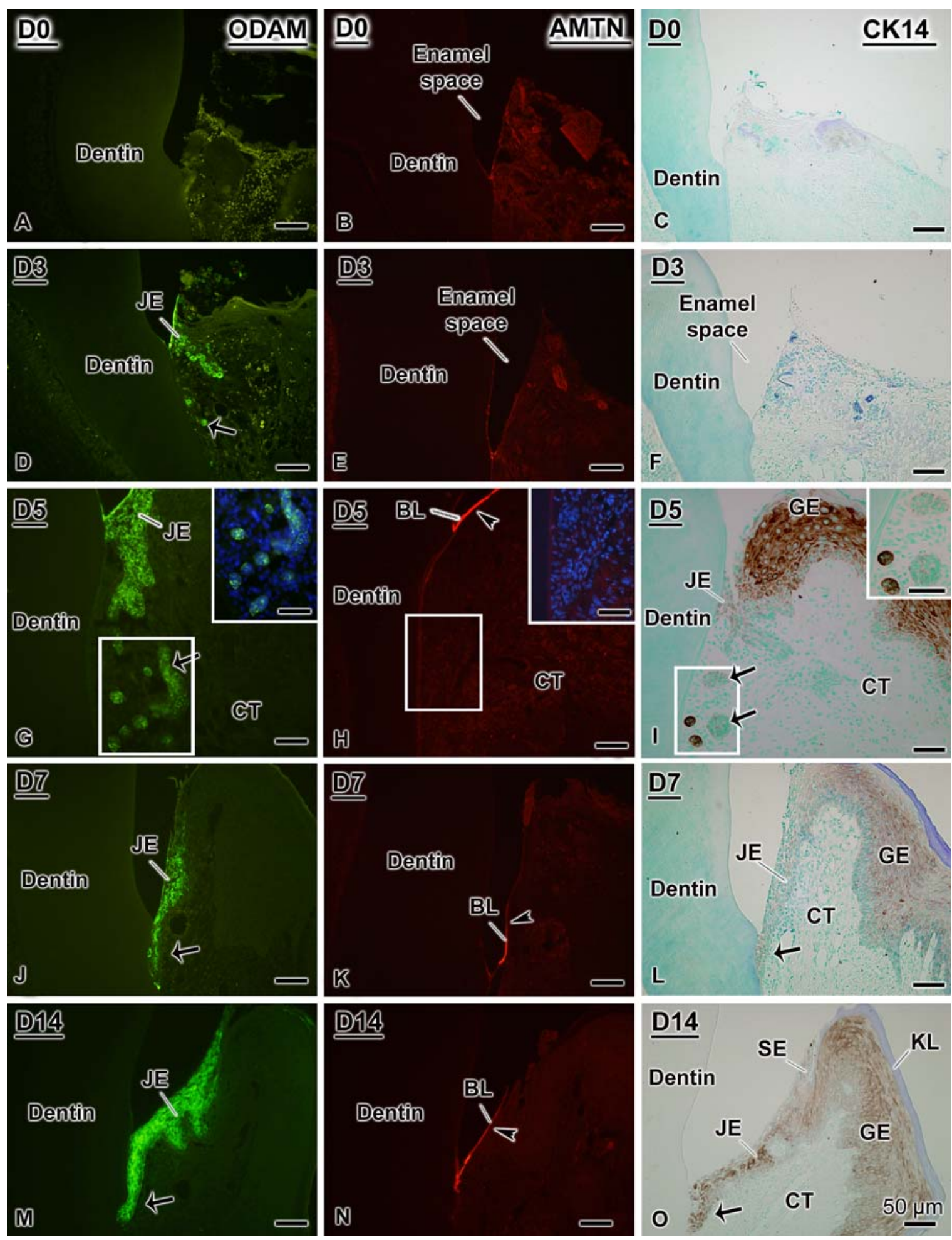

Fig. 3: Immunolabeling for ODAM (A,D,G,J,M), AMTN (B,E,H,K,N) and CK14 (C,F,I,L,O) at different time points following gingivectomy. No labeling for ODAM (A), AMTN (B) and CK14 (C) was seen in the residual tissues after surgery (D0). On day 3 (D3), ODAM (D) started to appear at the interface between the leading wound edge of the oral epithelium (OE) and some immunolabeled cell clusters (arrows) were seen in the underlying connective tissue (CT). However, no AMTN (E) and CK14 (F) were detected at the wound site. On day 5 (D5), ODAM (G) showed a more extensive cellular reaction in the regenerating JE and in cell clusters near the root surface in the subjacent periodontal tissue. Amelotin $(\mathbf{H})$ started to be observed at the cell-tooth interface (arrowhead), but no immunoreaction is seen in the cell clusters present in the CT (inset). Cytokeratin 14 (I) started to appear in the reforming gingival epithelium (GE) and weak to intense labeling was noted for cell clusters in the CT (inset). By day 7 (D7), ODAM (J) was present among the cells of the long JE (arrows) and at the interface with the enamel surface. Immunolabeling for CK14 (L) was present throughout the long JE (arrow) and in the GE. At day 14 (D14), despite the presence of a long JE, the immunolabeling for ODAM (M) and AMTN (N) was comparable to that seen in control samples (see Fig. 1G and H). Different from normal gingiva (see Fig. 1I), CK14 was detected in the entire cell layer of the regenerating GE, JE and sulcular epithelium (SE). BL, basal lamina; KL, keratinized layer. 

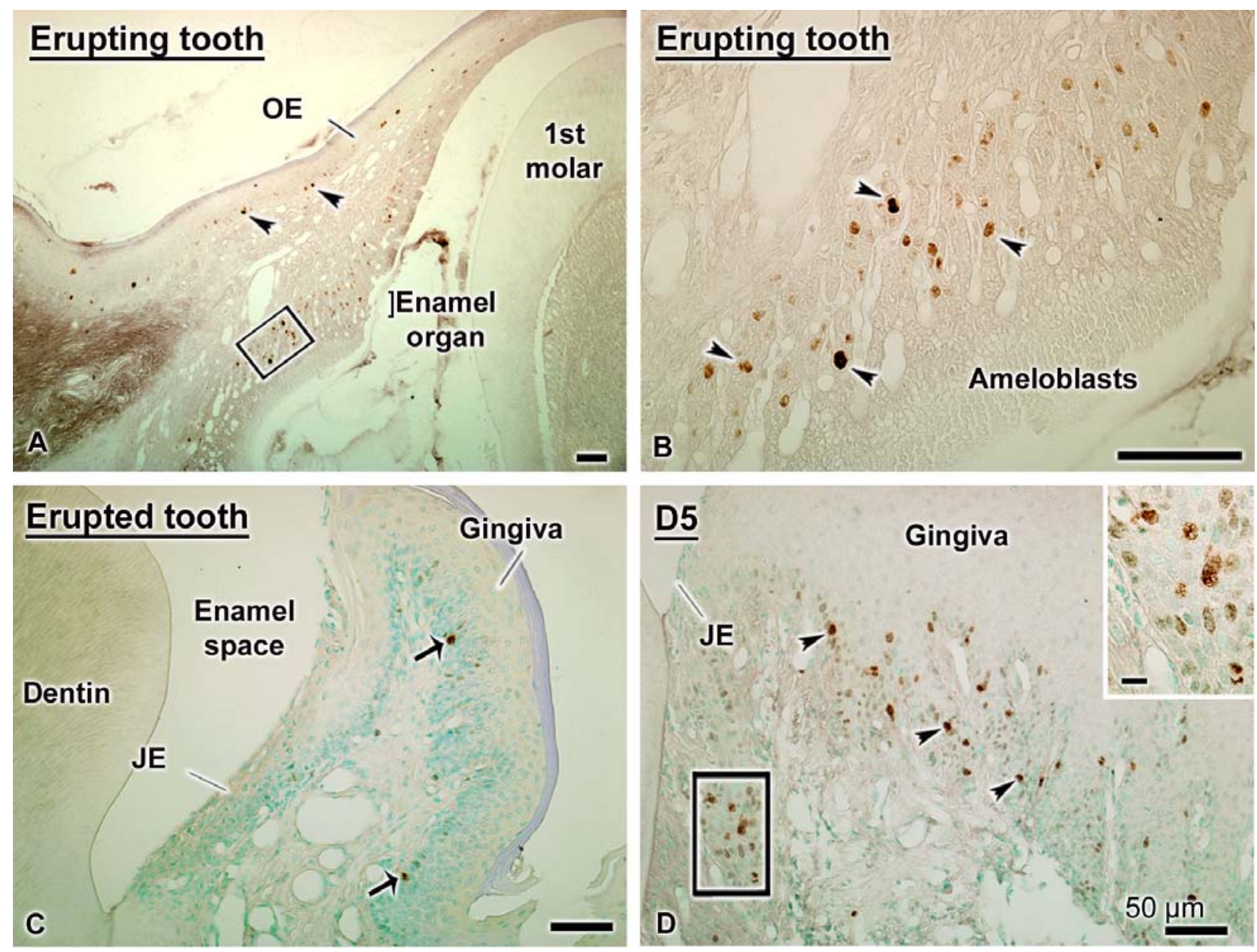

Fig. 4: Immunoperoxidase preparations for Ki67, a marker of cell proliferation (A-D) in erupting molars of 3 weeksold rat (A,B), in erupted tooth of adult rats (C) and at 5 days (D5) post-gingivectomy (D). (A) Several epithelial cells on the outer layer (boxed area) of the enamel organ and basal cells of the oral epithelium (OE, arrowheads) showed cell division activity. Picture in (B) is magnified region boxed in (A). (C) In fully-formed junctional epithelium (JE), basal cells (arrows) of the gingival epithelium (GE) were less frequently immunolabeled. The JE showed scarce or no stained cells. (D) At D5 post-surgery, the basal cells of the reforming gingiva (arrowheads) and cells of the clusters (inset) found in the subjacent connective tissue (CT), showed more proliferation activity than what is seen in the control sample.

JE showed very few or no labeled cells (Fig. 4C). Following gingivectomy and increasing from day 3 to day 5 post-surgery, basal cells of the reforming gingival epithelium, as well as epithelial clusters in the subgingival tissue, generally showed more stained cells (Fig. 4D) than that seen in the contralateral gingiva (not shown) or in the gingival epithelium of a normal erupted tooth (Fig. 4C).

\section{Control incubations}

Incubations with pre-immune antibodies and omission of primary antibody yielded no significant immunolabeling on sections of both treated and untreated samples (data not shown).

\section{Discussion}

This study describes for the first time the expression of ODAM and AMTN during formation and regeneration of the JE. ODAM was present at the level of the reducing enamel organ, and forming and regenerating JE. Both during tooth eruption and JE regeneration, AMTN was exclusively and discretely immunodetected at cell-tooth interfaces. Noteworthy, ODAM appeared during the initial phase of the healing process before AMTN and was associated both with cells and interfaces. We have also demonstrated that CK14 has a different expression pattern than ODAM and AMTN. Indeed, the cell-associated labeling for ODAM appears at high levels quite early in the process of gingival healing while that for CK14 attains a comparable intensity of expression only when the JE is regenerated. This suggests that ODAM is an early differentiation marker of the JE cell phenotype and that the presence of AMTN corresponds to establishment of an epithelial attachment to the tooth surface (Bosshardt and Lang, 2005; Schroeder, 1996).

ODAM was present in cell clusters along the tooth eruption front and in proximity to the regenerating JE. To our knowledge, such clusters have not been previously described, and whether they represent isolated structures 
or part of a network connected to the enamel organ or OE remains to be determined. However, their presence at these critical sites implicates them in early events leading to the formation of a JE. The fact that they express ODAM but not AMTN further suggests that ODAM represents an early marker of these cells and, as such, may be a determinant in their activity. During JE regeneration, these clusters exhibit variable labeling for the epithelial marker CK14, suggesting differences in cellular differentiation. While it is not possible from this study to determine whether the ODAM immunoreactive cell clusters along the tooth eruption front are related to those at the gingival wound edge, conceivably they may have analogous functions in establishing an epithelial seal around the tooth.

Prior to eruption of the tooth into the oral cavity, the enamel surface is covered by the reduced enamel organ. It has been proposed that this structure derives largely from the former stratum intermedium whose cells, unlike ameloblasts, maintain capacity to divide (Schroeder, 1996). However, in this study, as also previously described by Willberg et al. (2006), we demonstrate that in rat the cell division capacity is mainly associated with the outer aspect of the reduced enamel organ where the outer enamel epithelium is situated. It is thus likely that this apparently passive cell layer during tooth formation may actually have a delayed active role in establishment of the primary JE. This specialized epithelium is believed to normally regenerate into a secondary JE. Since the basal as well as the suprabasal cells of the JE have the ability to undergo cell division and turnover quite rapidly when minor gingival injury occurs, repair takes place from the JE itself (Salonen, 1994; Schroeder, 1996; Schroeder and Listgarten, 1997; Shimono et al., 2003; Ishikawa et al., 2005; Polimeni et al., 2006). However, when large portions of JE are surgically removed by gingivectomy, the reformation of the JE is believed to arise by repopulation from basal cells of the gingival epithelium (Schroeder and Listgarten, 1997; Bosshardt and Lang, 2005). While it cannot be ruled out that remnants of the JE following gingivectomy may contribute to regeneration, our study demonstrates that cells from the leading edge of the wounded gingiva, as well as clusters of epithelial cells expressing ODAM and situated within the periodontal space show high proliferation activity. This suggests that cells at both these sites are implicated in the regeneration of the JE. Indeed, during tooth root formation epithelial cells are believed to form a network in the periodontal space that connects to the JE (Spouge, 1984; Peters et al., 1995). In section, this network appears as clusters of cells called epithelial cell rests of Malassez that express CK14 (Rincon et al., 2006). Whether the clusters of cells expressing ODAM found within the periodontal space following gingivectomy derive from the wound edge or from epithelial cell rests of Malassez remains to be determined. It is widely believed that these cell rests of Malassez play a role in periodontal regeneration (Bosshardt, 2005; Rincon et al., 2006). We have recently reported that they do not normally express ODAM but dramatically do so at early times following disruption of periodontal integrity (Nishio et al., 2010). It is thus possible that ODAM could be part of the very initial events implicated in periodontal regeneration.

At this point we can only speculate as to the possible role of ODAM since there are no readily recognizable motifs within its sequence that would allow insights into potential functions. Its presence at multiple sites and its temporo-spatial distribution during JE formation and regeneration are somewhat reminiscent of the multifunctional matricellular proteins (MP). It should be pointed out here that the SCPP family of genes, which comprises Odam, originated by tandem duplication from a common ancestor - secreted protein acidic cysteine-richlike 1 (SPARCL1) gene - that initially arose from SPARC (Kawasaki et al., 2004), a well-known MP (Bornstein, 2009; Chiodoni et al., 2010). These proteins are secreted extracellularly where they interact with a variety of bioeffector molecules to modulate several cellular processes such as adhesion, migration, survival and proliferation (Bornstein, 2009; Chiodoni et al., 2010). The possibility that it may, similarly to MPs, participate in regulating tissue homoeostasis and inflammatory processes is also plausible. In this context, follicular dendritic cell secreted protein (FDC-SP), another protein whose gene is also part of the SCPP gene cluster just next to Odam (Marshall et al., 2002), is expressed in the JE (Shinomura et al., 2008) and periodontal ligament (Nakamura et al., 2005) where it may have a role in host defense. Finally, just like for several MPs (Chiodoni et al., 2010), the expression of ODAM is significantly upregulated during epithelial neoplastic transformation (Solomon et al., 2003; Aung et al., 2006; Kestler et al., 2008).

The delayed appearance of AMTN during the gingival wound healing when the regenerating JE reestablishes contact with the tooth, and its conspicuous localization in the area of the inner BL, suggest that this protein might be involved in events taking place at the JE-tooth interface (Moffatt et al., 2006b). The JE attaches to the tooth surface via the inner BL and it could be logically hypothesized that, as a component of the BL, AMTN contributes directly or indirectly by interacting with other components of the $\mathrm{BL}$, to the attachment mechanism of the JE (Moffatt et al., 2006b). ODAM could similarly be involved at the JE tooth interface (Moffatt et al., 2008). As such, it could be hypothesize that AMTN and perhaps also ODAM are part of the multimolecular epithelial attachment complex that binds the JE to the tooth surface. Other molecules of the same cluster such as FDC-SP that has been shown to bind to mineral (Shinomura et al., 2008) and, another member most recently identified, secretory calcium-binding phosphoprotein proline-glutamine rich 1 (SCPPPQ1 / EO463) (Moffatt et al., 2006a; Kawasaki, 2009) could also be potential partners for such interactions.

In conclusion, the data suggest that both ODAM and AMTN are implicated in the formation and regeneration of the JE. ODAM appears to have a distinctive expression profile and to be multifunctional. It may influence the cellular events during the apical extension of the gingival wound edge to reestablish a functional JE, and as AMTN, participate in the formation of the attachment complex of JE to the tooth surface. Its upregulation in certain epithelial 
neoplasms and in epithelial cell rests of Malassez following periodontal challenge, and its normal expression by the JE whose cells are incompletely differentiated, further suggest that ODAM may play a role in modulating the cell status. This is consistent with the observation that ODAM expressing cells at the leading wound edge and in the cell clusters in the periodontium have a higher cell proliferation activity.

\section{Acknowledgements}

Dr. Shingo Kuroda is an Associate Professor in the Department of Orthodontics and Dentofacial Orthopedics at The University of Tokushima Graduate School of Oral Sciences, Japan, and a visiting researcher at the Laboratory for the Study of Calcified Tissues and Biomaterials, Faculty of Dentistry, Université de Montréal, Canada. We extend our thanks to Ms Cynthia Török for technical assistance. This study was supported by the Department of Foreign Affairs and International Trade, Government of Canada, and by the Canadian Institutes of Health Research. Pierre Moffatt is supported by the Shriners of North America.

\section{References}

Abe Y, Hara Y, Kato I (1995) Histological study of lectin binding in regenerated rat junctional epithelium. $\mathrm{J}$ Periodontal Res 30: 238-244.

Aung PP, Oue N, Mitani Y, Nakayama H, Yoshida K, Noguchi T, Bosserhoff AK, Yasui W (2006) Systematic search for gastric cancer-specific genes based on SAGE data: melanoma inhibitory activity and matrix metalloproteinase-10 are novel prognostic factors in patients with gastric cancer. Oncogene 25: 2546-2557.

Bornstein P (2009) Matricellular proteins: an overview. J Cell Commun Signal 3: 163-165.

Bosshardt DD (2005) Are cementoblasts a subpopulation of osteoblasts or a unique phenotype? J Dent Res 84: 390-406.

Bosshardt DD, Lang NP (2005) The junctional epithelium: from health to disease. J Dent Res 84: 9-20.

Chiodoni C, Colombo MP, Sangaletti S (2010) Matricellular proteins: from homeostasis to inflammation, cancer, and metastasis. Cancer Metastasis Rev 29: 295307.

Endl E, Gerdes J (2000) The Ki-67 protein: fascinating forms and an unknown function. Exp Cell Res 257: 231237.

Hormia M, Owaribe K, Virtanen I (2001) The dentoepithelial junction: cell adhesion by type I hemidesmosomes in the absence of a true basal lamina. $\mathrm{J}$ Periodontol 72: 788-797.

Hormia M, Virtanen I, Quaranta V (1992) Immunolocalization of integrin $\mathrm{a}_{6} \mathrm{~b}_{4}$ in mouse junctional epithelium suggests an anchoring function to both the internal and the external basal lamina. J Dent Res 71: 15031508

Huq NL, Cross KJ, Ung M, Reynolds EC (2005) A review of protein structure and gene organisation for proteins associated with mineralised tissue and calcium phosphate stabilisation encoded on human chromosome 4. Arch Oral Biol 50: 599-609.

Ishikawa H, Hashimoto S, Tanno M, Ishikawa T, Tanaka T, Shimono M (2005) Cytoskeleton and surface structures of cells directly attached to the tooth in the rat junctional epithelium. J Periodontal Res 40: 354-363.

Iwasaki K, Bajenova E, Somogyi-Ganss E, Miller M, Nguyen V, Nourkeyhani H, Gao Y, Wendel M, Ganss B (2005) Amelotin - a novel secreted, ameloblast-specific protein. J Dent Res 84: 1127-1132.

Kawasaki K (2009) The SCPP gene repertoire in bony vertebrates and graded differences in mineralized tissues. Dev Genes Evol 219: 147-157.

Kawasaki K, Weiss KM (2003) Mineralized tissue and vertebrate evolution: the secretory calcium-binding phosphoprotein gene cluster. Proc Natl Acad Sci USA 100: 4060-4065.

Kawasaki K, Weiss KM (2006) Evolutionary genetics of vertebrate tissue mineralization: the origin and evolution of the secretory calcium-binding phosphoprotein family. J Exp Zool B Mol Dev Evol 306: 295-316.

Kawasaki K, Suzuki T, Weiss KM (2004) Genetic basis for the evolution of vertebrate mineralized tissue. Proc Natl Acad Sci USA 101: 11356-11361.

Kestler DP, Foster JS, Macy SD, Murphy CL, Weiss DT, Solomon A (2008) Expression of odontogenic ameloblast-associated protein (ODAM) in dental and other epithelial neoplasms. Mol Med 14: 318-326.

Mackenzie IC (1987) Nature and mechanisms of regeneration of the junctional epithelial phenotype. J Periodontal Res 22: 243-245

Marshall AJ, Du Q, Draves KE, Shikishima Y, HayGlass KT, Clark EA (2002) FDC-SP, a novel secreted protein expressed by follicular dendritic cells. J Immunol 169: $2381-2389$.

Masaoka T, Hashimoto S, Kinumatsu T, Muramatsu T, Jung HS, Yamada S, Shimono M (2009) Immunolocalization of laminin and integrin in regenerating junctional epithelium of mice after gingivectomy. J Periodontal Res 44: 489-495.

Moffatt P, Smith CE, Sooknanan R, St-Arnaud R, Nanci A (2006a) Identification of secreted and membrane proteins in the rat incisor enamel organ using a signal-trap screening approach. Eur J Oral Sci 114 Suppl 1: 139-146.

Moffatt P, Smith CE, St-Arnaud R, Simmons D, Wright JT, Nanci A (2006b) Cloning of rat amelotin and localization of the protein to the basal lamina of maturation stage ameloblasts and junctional epithelium. Biochem $\mathrm{J}$ 399: 37-46.

Moffatt P, Smith CE, St Arnaud R, Nanci A (2008) Characterization of Apin, a secreted protein highly expressed in tooth-associated epithelia. J Cell Biochem 103: $941-956$.

Nakamura S, Terashima T, Yoshida T, Iseki S, Takano Y, Ishikawa I, Shinomura T (2005) Identification of genes preferentially expressed in periodontal ligament: specific expression of a novel secreted protein, FDC-SP. Biochem Biophys Res Commun 338: 1197-1203.

Nanci A, Wazen RM, Zalzal S, Fortin M, Goldberg HA, Hunter GK, Ghitescu DL (2004) A tracer study with 
systemically and locally administered dinitrophenylated osteopontin. J Histochem Cytochem 52: 1591-1600.

Nishio C, Wazen R, Kuroda S, Moffatt P, Nanci A (2010) Disruption of periodontal integrity induces expression of apin by epithelial cell rests of Malassez. J Periodontal Res 45: 709-713.

Park JC, Park JT, Son HH, Kim HJ, Jeong MJ, Lee CS, Dey R, Cho MI (2007) The amyloid protein APin is highly expressed during enamel mineralization and maturation in rat incisors. Eur J Oral Sci 115: 153-160.

Peters BH, Peters JM, Kuhn C, Zoller J, Franke WW (1995) Maintenance of cell-type-specific cytoskeletal character in epithelial cells out of epithelial context: cytokeratins and other cytoskeletal proteins in the rests of Malassez of the periodontal ligament. Differentiation 59: 113-126.

Polimeni G, Xiropaidis AV, Wikesjo UM (2006) Biology and principles of periodontal wound healing/ regeneration. Periodontol 2000 41: 30-47.

Rincon JC, Young WG, Bartold PM (2006) The epithelial cell rests of Malassez - a role in periodontal regeneration? J Periodontal Res 41: 245-252..

Sabag N, Mery C, Garcia M, Vasquez V, Cueto V (1984) Epithelial reattachment after gingivectomy in the rat. J Periodontol 55: 135-141.

Salonen JI (1994) Proliferative potential of the attached cells of human junctional epithelium. J Periodontal Res 29: 41-45.

Schroeder HE (1991) The rate of the eruption of human teeth: A review. Schweiz Monatsschr Zahnmed 101: 279284.

Schroeder HE (1996) The junctional epithelium: origin, structure and significance. Acta Med Dent Helv 1: 155 167.

Schroeder HE, Listgarten MA (1997) The gingival tissues: The architecture of peridontal protection. Periodontol 2000 13: 91-120.

Schroeder HE, Listgarten MA (2003) The junctional epithelium: from strength to defense. J Dent Res 82: 158161.

Shimono M, Ishikawa T, Enokiya Y, Muramatsu T, Matsuzaka K, Inoue T, Abiko Y, Yamaza T, Kido MA, Tanaka T, Hashimoto S (2003) Biological characteristics of the junctional epithelium. J Electron Microsc 52: $627-$ 639.

Shinomura T, Nakamura S, Ito K, Shirasawa S, Hook M, Kimura JH (2008) Adsorption of follicular dendritic cell-secreted protein (FDC-SP) onto mineral deposits. Application of a new stable gene expression system. J Biol Chem 283: 33658-33664.

Siddiqui S, Bruker CT, Kestler DP, Foster JS, Gray KD, Solomon A, Bell JL (2009) Odontogenic ameloblast associated protein as a novel biomarker for human breast cancer. Am Surg 75: 769-775.

Solomon A, Murphy CL, Weaver K, Weiss DT, Hrncic R, Eulitz M, Donnell RL, Sletten K, Westermark G, Westermark P (2003) Calcifying epithelial odontogenic (Pindborg) tumor-associated amyloid consists of a novel human protein. J Lab Clin Med 142: 348-355.

Spouge JD (1984) The rests of Malassez and chronic marginal periodontitis. J Clin Periodontol 11: 340-347.
Takata T, Nikai H, Ijuhin N, Okamoto H (1986) Ultrastructure of regenerated junctional epithelium after surgery of the rat molar gingiva. J Periodontol 57: 776783.

Tsuchiya Y, Muramatsu T, Masaoka T, Hashimoto S, Shimono M (2009) Effect of the dental adhesive, 4-META/ MMA-TBB resin, on adhesion and keratinization of regenerating oral epithelium. J Periodontal Res 44: 496502.

Warshawsky H, Moore G (1967) A technique for the fixation and decalcification of rat incisors for electron microscopy. J Histochem Cytochem 15: 542-549.

Willberg J, Syrjanen S, Hormia M (2006) Junctional epithelium in rats is characterized by slow cell proliferation. J Periodontol 77: 840-846.

\section{Discussion with Reviewers}

Reviewer I: What could be the reason why two products of cells of the ameloblast lineage are again formed, when the junctional epithelium regenerates from the oral epithelium which normally does not express ODAM and AMTN? Could there be instructive signals from the periodontal connective tissue?

Authors: At this point, the exact mechanism that leads to the expression of these two proteins is still not completely understood. Yes, the connective tissue underlying the JE may be a potential 'instructor' for the expression of JE products. Indeed, this connective tissue has been shown to be functionally different from that underneath the rest of the oral epithelium (Mackenzie, 1987, text reference). In a recent paper, we have shown that disruption of periodontal integrity, including by gingivectomy, will induce the expression of ODAM by epithelial cell rests of Malassez present along the root (Nishio et al., 2010, text reference). This suggests that changes in the environment either exert a direct effect on the cell rests or may alternatively induce a response from connective tissue components that may provide instructive signaling. Cells at the leading gingival wound edge may be similarly influenced. In addition, since AMTN is expressed at a later time point, the presence of a mineralized tooth surface may also provide a 'cue' to induce its expression in the inner basal lamina of the JE, thus leading to establishment of a functional JE.

Reviewer II: Could the JE be a likely source for epithelial dental stem cells? Since the tissue can regenerate it could be harvested to some extent, and the question is how much of a dental tissue is the JE? There seem to be some similarities between the epithelial cell rests of Malassez (ERM) and the JE, and during the last TMD meeting in Berlin there was a really interesting talk on the possibility of isolating epithelial stem cells from the ERM as a possible source of stem cells. How close would the JE be to the dental fate, and could it be used as a source for epithelial dental stem cells?

Authors: To our knowledge, no studies have been reported on the use of the JE as a potential source of dental stem cells. However, since it is possible to 'dedifferentiate' in 
vitro epithelial cells, it would be very interesting to investigate whether incompletely-differentiated cells of the JE can be exploited as a source of stem cells and whether they have a dental specificity. The recent report that stem cells can be obtained from ERM and the fact that ERM are believed to form a network that is in continuity with the JE lend certainly support to this possibility.

Reviewer II: Would adding an enriched population of ODAM expressing cells speed up JE regeneration?

Authors: Since expression of ODAM is an early event in JE regeneration, it could be conceived that adding the protein or cells that express ODAM, or locally overexpress the protein using gene transfer could indeed influence the process. Since little is known at this time about ODAM, determining whether ODAM has any such therapeutic potential will have to await further experimentation.

Reviewer II: How could we elucidate the exact constitution of the JE compartment? How many cell populations would there be in the JE? What functions do they perform? It seems to be a current general problem for many dental compartments at the moment, whether you look at the JE, cervical loop area, HERS or any mesenchymal compartment. A microarray could give us elevated and downregulated genes. They would still need to be validated.

Authors: A recent paper by Hayashi et al. (2010) has combined laser microdissection and microarray analysis to obtain an overview of the gene expressed in the JE and to search for specific genetic markers. This approach may help address the question. The fact that all cell layers of the JE can divide (Schroeder and Listgarten, 1997, text reference) and that ODAM seems to be expressed throughout the JE, suggests a certain cellular uniformity, at least in a well-developed JE.
Reviewer II: Would it be useful to work on elucidating the functional dental compartments such as the JE? And should we as a community put more joint effort into these projects that could be of general importance?

Authors: The JE is the first line of defense against periodontal disease, which in its various forms affects over $80 \%$ of the population (Burt, 2005). Clearly, this unique compartment merits the attention of our scientific community. The association of some of the JE proteins with cancer and the fact that the tooth is a source of stem cells further highlight the general importance of better understanding dental compartments.

Reviewer III: Is there any difference in ODAM and AMTN expression between the new and the mature junctional epithelium as these are believe to be of either enamel organ or gingival origin?

Authors: We have not observed any significant difference in expression pattern of AMTN and ODAM in the established primary and regenerated JE, as well as any readily apparent structural differences between them. One difference may be that the primary JE forms from cells that are already expressing both proteins while during regeneration there is a temporal difference in expression that correlates with the histological progression in reformation.

\section{Additional References}

Burt B (2005) Position paper: Epidemiology of periodontal diseases. J Periodontol 76: 1406-1419.

Hayashi Y, Matsunaga T, Yamamoto G, Nishii K, Usui M, Yamamoto M, Tachikawa T (2010) Comprehensive analysis of gene expression in the junctional epithelium by laser microdissection and microarray analysis. J Periodontal Res 45: 618-625. 